\title{
Anterior Cruciate Reconstruction Using Bone-patellar Ligament - Bone Autograft
}

\author{
L.N. Gakuu, MBChB, MMed (Surg), FCS (ECSA), Associate Professor, Department of Orthopaedic Surgery, \\ College of Health Sciences, University of Nairobi, P.O. Box 55164-00200, Nairobi, Kenya
}

\begin{abstract}
Background: Injuries to ligaments of knee are common in our society especially in the young athletes and sports men participating in football, volleyball, rugby etc. Tears of the anterior cruciate ligament $(A C L)$ leads to knee instability which leads to inability to participate in sports. In the none athletes ACL injury tends to decline in their level of daily activity. This is a study of ACL tears seen and managed by the author over a long period along with scientific data of clinical presentations, diagnosis, associated injuries, the ACL repair, rehabilitation protocol and the overall outcome.
\end{abstract}

Objective: To review the authors experience of patients with anterior cruciate ligament tears who have been treated by bone-patellar tendon-bone autografts. That was to be achieved through clinical, radiological, athroscopy and extensive rehabilitation programme and arthroscopic review after one year follow-up.

Setting: Menelik Hospital and the Nairobi Hospital.

Methods: Thirty five patients with anterior cruciate ligament tear in one knee and a normal contralateral knee were evaluated and followed up for six years. Clinical history, physical evaluation, $X$-ray and athroscopies were done to rule out concomitant meniscal lesions and facilitate their treatment accurately. Follow up arthroscopy; physical examinations by Lachman test, pivot test and anterior drawer test were done. Subjective and objective data were obtained and reviewed to assess final outcome.

Results: A total of 35 patients were seen and treated. Only 25 patients were available having been followed up fully. There were 18 males and seven females giving a male to female ratio of 2:1. The injuries were unilateral $18(72 \%)$ on the right and seven ( $28 \%)$ on the left. Initial Lachman"s test was graded at 2 and 3 while anterior drawers test and pivot test at 2 and 3 respectively in all patients. Post operatively these improved to grade zero with negative pivot test in all patients. The subjective results were excellent and good in $80 \%$ of the patients, while the objective were $68 \%$. Follow up arthroscopy showed a stable ligament at one year while follow up X-ray showed mild arthrosis. A few complications occurred with one infection and calcification of ligament in two patients, arthrofibrosis in two patients.

Conclusion: This study is of clinical relevance as it shows alleviation of pain after surgical treatment of the torn ACL which has been described as the stabiliser of the knee and guardian of the Meniscus.

\section{INTRODUCTION}

The anterior cruciate ligament $(\mathrm{ACL})$ of the knee is a primary anterior rotatory stabiliser of the joint $(1,2)$. $\mathrm{ACL}$ insufficiency can lead to degenerative arthritis $(3,4)$ and functional instability and a high incidence of meniscal tears (5). The relatively poor results obtained by none surgical treatment of torn $\mathrm{ACL}(6,7)$ have led to orthopaedic surgeons to repair or reconstruct torn $\mathrm{ACL}$. The most common methods of reconstruction of $\mathrm{ACL}$ use a bone-patellar tendon-bone graft (8) or one derived from hamstring tendons, while allografts and artificial ligaments have been used since 1980 (9). Outcomes following $\mathrm{ACL}$ repair are generally good (10-12).

The mid third patella-bone tendon auto graft is often preferred for use because the bone to bone attachment sites provide rapid healing and insertional strength. A $10 \mathrm{~mm}$ slit of the patellar tendon has been found to be $104 \%$ as strong as normal (13) ACL ligament. 


\section{MATERIALS AND METHODS}

Between 1999 and 2004, thirty five arthroscopicaly assisted and occasional miniarthrotomy $\mathrm{ACL}$ reconstructions were done. The middle third of the bone-patellar tendon-bone (BTB) autografts was used. Patients ages ranged from 18 to 54 years with an average of 36 years. There were 8 males and 7 females giving a male to female ratio of 2:1. Ten patients were lost to follow up before minimum of two years follow up. The average follow up time was four years (maximum 6 years) $15(60 \%)$ of the patients had sports related injuries due to football (4), volleyball (2), basketball (3), rugby (2), cricket (2), lawn tennis (2). The rest 10 (40\%) were due to falls and road traffic accidents (2).

Eight (32\%) of the patients had associated injuries which included meniscal tear (4), loose bodies (4), degenerative changes (4) chondro malacia patellae, and one chondral fracture of the lateral femoral condyle. In 10 patients reconstruction was done within 10 weeks while 15 were done after two months

Follow up examination included physical examination, an $\mathrm{X}$ ray and second review arthroscopy at approximately one year.

Surgery: The ACL reconstructions were performed using arthroscopic assisted technique and occasionally in difficult cases a mini arthrotomy was done (14). The operations were under general anaesthesia $15(60 \%)$ and spinal anaesthesia $10(40 \%)$ all under tourniquet control. The grafts were harvested from the middle third of the patellar ligament using an oscillating saw to incorporate a piece of bone from the patellar proximally and from the tibial tubercle distaly. The surgical technique was transitibial-transcondlar single entry technique using a guide under arthroscopic control. Additionally in later cases $18(72 \%)$ notchplasty was done superiorly and laterally to widen and deepen the intercondylar notch to avoid impingement of the graft.

Tension was applied to the graft with knee flexed at 30 degrees until anteroposterior instability could not be elicited by Lachman test (15). The femoral and tibial ends were then secured using cancellous interference screws.

Post-operative regime: Initially 1999-2001 the patients were given a plaster casts for four weeks and mobilised on crutches followed by physiotherapy up to three months when resisted exercises were started. Thereafter (2002-2004) the regime changed whereby the patient was allowed immediate motion and progressive resistance exercises and full weight bearing started simultaneously on the second day (16). The patients remained in hospital for five days to one week for supervised physiotherapy and observations to rule out infections or deep vein thrombosis. Thereafter they were discharged for outpatient physiotherapy.

The pre and post operative assessment included Lachman's test (14), pivot test (17) and the anterior drawers test. Follow up was done at three, six, twelve months and every year thereafter with follow up arthroscopy at one year in $15(60 \%)$ of the patients.

\section{RESULTS}

All the injuries were unilateral $18(72 \%)$ on the right and seven $(28 \%)$ on the left. The mean time from injury to attendance at the clinic was 14 months (two months to five years). After the first injury most patients 20 (80\%) had suffered repeat episodes of instability, locking of the knee and effusions.

A preliminary arthroscopy showed medial meniscal tears in two and lateral meniscal tear in another two patients. Lachman's test (14) was performed at 30 degrees of knee flexion while anterior drawers test (16) was done at 70 degrees. Preoperatively the Lachman's test was graded 2 to 3 with a soft endpoint while the anterior drawers test and pivot shift were graded at 2 to 3 respectively in all patients. Postoperatively the Lachman's test and Anterior drawers test improved to grade 0 to 1 with a firm end point. The pivot test remained negative in all knees post-operatively.

The objective assessment was done considering the normal knee movements as normal - lack of extension less than 3 degrees, flexion less than 5 degrees, nearly normal- lack of extension 3-5 degrees, flexion 6-15 degrees, abnormal lack of extension 6-10 degrees, flexion 16-25 degrees and severely abnormal much greater deficiency (Table 1).

The subjective results are shown in Table 1 after evaluation of the symptoms were assessed as normal - absence of pain, swelling, and giving way; nearly normal (ability to perform only light activity without symptoms) and severely abnormal (no activity possible without symptoms).

One patient showed mild valgus laxity after sliding on a wet ground postoperatively. Pain free crepitus of patellofemoral joint was noted in two (8\%) of the patients and medial compartment three (12\%) four patients experienced slight difficulties in kneeling. 
Table 1

\begin{tabular}{lcc}
\hline & Subjective assessment & Objective assessment \\
\hline Normal & 15 & 13 \\
Nearly normal & 5 & 4 \\
Abnormal & 3 & 6 \\
Severely abnormal & 2 & 2 \\
\hline Total & 25 & 25 \\
\hline
\end{tabular}

Quadriceps wasting measured at 10 centimetres above superior pole of the patella was noted in three (12\%) despite physiotherapy. Anterior knee pain was noted in three (12\%) patients.

Radiological assessment: Postoperatively mild to moderate osteoarthrosis (19) was seen as narrowing of the joint space in four knees (16\%) which incidentally had meniscal injuries. Radiological osteo arthrosis was noted initially on the third postoperative year but no pains. A lateral X-ray was taken with knee in full extension showed the anterior border of most of tibial tunnels lay posterior to the apex and the roof of the intercondylarnotch, indicating no impingment (20).

Arthroscopic assessment: Of the 15 patients who underwent second look arthroscopy, approximately one year postoperative 18 (72\%) had fully functioning knee. The probe test confirmed a stable ligament while two ligaments were noted to have calcifications at the base. There were three patients with arthrofibrosis which were debrided satisfactorily at arthroscopy. Chondromalacia patellae as described by Outerbridge (21) was seen in four patients.

Complications: Post-operatively there was one wound infection, two deep venous thrombosis (DVT), two post operative haematomas. One fracture of the lateral femoral condyle occurred in a patient who fell in the bathroom.

\section{DISCUSSION}

The present study was based on investigation of a small number of patients whose ACLs were reconstructed with bone-patellar ligament- bone (BTB) autografts and who were followed up to six years. The study correlates improvement in surgical technique and change in the rehabilitation protocol. The overall satisfactory results (good to excellent) was achieved in 20 (80\%) of the patients. During the course of the study changes in surgical technique and postoperative rehabilitation are noted. At the beginning isometric graft replacement and notchplasty were not adhered to strictly but as the study progressed the indication became clear. The notchplasty creates a larger notch superiorly and laterally thus allowing good visualisation posteriorly to enable identify the proper tunnel placement. This alleviates graft impingement and its associated graft fraying and limitation of extension $(20,22)$. The rehabilitation protocol has also changed considerably, between 19992002. The patients were cast immobilied for four weeks and only allowed active weight bearing at three months with resisted exercises and knee extension. From 2003 onwards the rehabilitation protocol changed to a more aggressive early mobiliation, from day two with weight bearing as soon as patients are comfortable (16). This aggressive early mobiliation has not proven harmful in any way (23). The review of the literature by Gillquist (24) found the achievements of "good to excellent" results in ACL reconstruction to be the order of $66 \%$ to $90 \%$. This compares well with our patients who achieved $80 \%$. No failure of the ligament occurred as opposed to reported failure rates of $5 \%-15 \%$ for autografts at three to five years after surgery (26).

On subjective assessment $20(80 \%)$ of our patients were satisfied with the outcome which is comparable to other series in the literature $(27,28)$. Most had returned to work within three months.

The passage of time from injury to surgery (two months to five years) was rather long and could have increased the incidence of meniscal and chondral damage as seen in 13 (52\%) patients at arthroscopy and as per radiological findings (29). Theoretically there maybe a proprioception advantage in conserving the $A C L$ remnants at surgery though this takes too long to recover (30). Until this occurs the stability of knee will depend on an intact anterior cruciate ligament which must be isometricaly placed, well secured and free from impingement $(21,22)$. Twenty two $(88 \%)$ of our 
patients confirmed a good recovery of the quadriceps and hamstring after surgery while only three had persisted weak quadriceps despite physiotherapy. Our incidence of patelo femoral crepitus was $8 \%$ compared with reports of $45 \%$ in the literature (31). Follow-up Xray did not show any tunnel expansion as reported in literature of up to $54 \%$ (32).

The occurrence of postoperative anterior knee pain after patella tendon autograft has been widely reported in the literature $(33,34)$. In this study only three patients reported severe arterior knee pain. Shellborne and Trumper (34) suggested and recommended early full extension of the knee to avoid this problem which was done in our accelerated rehabilitation programme. Results of this study indicate subjective and objective measurements are slightly different and this could be attributed to the residual complaints of knee pains by patients whose injuries occurred at work for compensation purposes.

\section{CONCLUSION}

This study shows that arthroscopically assisted $A C L$ reconstruction using bone-patellar ligament, bone autografts and mini-arthrotomy can be done successfully. The use of autografts allows anatomical alignment and stability of the knee joint thereby avoiding complications of an unstable knee. The techniques of autograft harvesting and fixation have changed with time and so has the rehabilitation protocols. Our patients tend to come late and we suggest community health awareness especially in schools and sports clubs to create awareness for early diagnosis and management.

\section{REFERENCES}

1. Hawkins R.J. Misamore G.W and Merrit T.R. Follow up of the none operated anterior cruciate ligament tear. Amer. J. Sports Med. 1986; 14: 205.

2. Kieffer D.A., Curnow R.J. and SouthWell R.B. Anterior Cruciate Ligament arthroplasty. Amer. J. Sports Med. 1984; 12: 301.

3. Rovere G.D. and Adair D.M. Anterior Cruciate Deficient Knees. A review of the literature. Amer. J. Sports Med. 1983; 11: 412 .

4. Kennedy J.C. and Fowler P.J. Medial and arterior instability of the knee. An anatomical clinical study using stress machines. J. Bone Joint Surg. 1971; 53: 1257.
5. Reider B. and Marshall J.L. Anterior cruciate guardian of the meniscus. Orthop. Rev. 1979; 8: 83.

6. Marzo J.M and Warren R.I. Results of non-operative treatment of anterior cruciate ligament injury. Adv. Orthop. Surg. 1991; 15: 59

7. Feagin J.A and Curl W.W. The isolated tear of the anterior cruciate ligament: A five-year follow-up. J. Bone Joints Surg. 1972; 54A: 1340

8. Johnson R.J., Beynnon B.D., Nichols C.E., et al. The treatment of injuries of the anterior cruciate ligament. J. Bone Joint Surg. 1992; 74A: 140-151.

9. Greisse P.E. and Steadman J.R. Revision of failed prosthetic anterior cruciate ligament reconstruction. Clin. Orthrop. 1996; 325: 78-90.

10. Freedman K.B., D'Amato M.J., Nedeff D.D., et al. Arthroscopic anterior cruciate ligament reconstruction and a meta-analysis comparing pattelar tendon and hamstring tendon autograft. Amer. J. sports Med. 2003; 31:2-11.

11. Yunes M., Richards J.C., Engels E.A., et al. Patela versus hamstring tendon in anterior cruciate ligament reconstruction: A meta- analysis. Arthroscopy. 2001; 17: 248-257.

12. Anderson A.F., Snydez R.B. and Lipsimb. Anterior cruciate ligament reconstruction. A prospective randomied study of three surgical methods. Amer. J. Sports Med. 2001; 29: 272-279.

13. Oensten M., Lysholm J. and Gillquist J. Suture of fresh ruptures of the anterior cruciate ligament. A five-year follow-up. Acta. Orthop. Scan. 1984; 55: 270.

14. Al-Zaharini S., Franceschi J.P., Loste J., et al. Anterior cruciate ligament reconstruction by miniarthrotomy. Int Orthop. (SICOT). 21: 161-163.

15. Torg J., Conrad W. and Kalen V. Clinical diagnosis of ACL instability in the athlete. Amer. J. Sports Med. 1976; 4: 84-93.

16. Shelbourne K.D. and Gray T. Anterior cruciate ligament reconstruction with autogenous patellar tendon graft followed by accelerated rehabilitation: A two to nine-year follow-up. Amer. J. Sports Med. 1997; 25: 786-795.

17. Galaway R.D. and Becipre A. Pivot shift: A clinical sign of symptomatic ACL Insufficiency. J. Bone Joint Surg. (Brit) 1972; 54-b: 763-764.

18. Larrie P., Fletcher J. and Duran V. Patient satisfaction needs as related to knee stability and objective findings after $\mathrm{ACL}$ reconstruction using LARS artificial ligament. The Knee. 2000; 7: 157-163.

19. Ahlback S., Osteoarthritis of the knee: A radiographic investigation. Acta. Radiol. Diagn. 1968; Sppl 277: 7-72. 
20. Howel S.M. and Taylor M.A. Failure of reconstruction of the anterior cruciate ligament due to impingement by the intercondylar roof. J. Bone Joint Surg. (Amer). 1993.

21. Outerbridge R.E. The etiology of chondromalacia patellae. J. Bone Joint Surg. (Brit). 1961; 8: 752-757.

22. Barry M., Kong K.C., Thomas R., et al. Instrumentation to avoid impingement lesions in anterior crucial ligament reconstruction. The Knee. 1996; 3: 191-197.

23. Noyes F.R. and Margine R.F. Anterior cruciate ligament reconstruction. Amer. J. Sports Med. 1987; 15: 149.

24. GillQuist J. Repair and reconstruction of the ACL: Is it good enough? Arthroscopy. 1993; 9: 68-71.

25. Hackenbruch W., Hey W. and Henche H.R. Late results after $A C L$ reconstruction with an autologous free patellar tendon graft. In Jacob R.P. and Stauli H.V. eds. The knee and cruciate ligaments; Berlin etc. Springer - Verlag. 1990; 112-115.

26. Shellbourne K.D., Whitaker H.J., McCarrol J.R., et al. Anterior cruciate ligament injury. Evaluation of intra-articular reconstruction of acute tear without repair: Two to seventeen-year follow-up of 155 athletes. Amer. J. Sports Med. 1990; 18: 484-488.

27. Roos E.M., Roos H.P., Lohmander L., et al. Knee injury and Osteoarthrosis outcome score (KOOS). Development of a self-administered outcome measure. J. Orthop. Sports Phys. Ther. 1998; 28: 86-96.

28. Larrie P., Fletcher J. and Duran N. Patient satifaction needs and related to knee stability and objective findings after $\mathrm{ACL}$ reconstruction using LARS artificial ligament. Knee. 2000; 7: 157-163.

29. Sharif K., Yianna Kopoulos C., Fute P., et al. Progressive comorbidity in the knees of patients with chronic instability awaiting anterior cruciate ligament reconstruction. J. Bone Joint Surg. (Brit). 2005; 87B Supplement 11: 147.

30. Fremery R.W., Loben Hoffer P., Zeicher J., et al. Proprioception after rehabilitation and reconstruction in knees with deficiency of the anterior cruciate ligament: A prospective longtudinal study. J. Bone Joint Surg. (Brit). 200?; 82-B: 801-861.

31. Arthroscopically assisted anterior cruciate ligament reconstruction using patella tendon autograft: Five to nine-year follow-up evaluation. Amer. J. Sports Med. 1998; 26: 20-29.

32. Noyes F.R. and Barber-Westin S.P. Reconstruction of the anterior cruciate ligament with human allografts: Comparison of early and later results. J. Bone Joint Surg. (Amer). 1996; 78-A: 524-537.

33. Back B.R., Tradonsky S., Bojchuk J., et al. Arthroscopically assisted anterior cruciate ligament reconstruction using patella tendon autograft. Amer. J. Sports Med. 1998; 26: 20-29.

34. Shelbourne K.D. and Trumper R.V. Preventing anterior knee pains after anterior cruciate ligament reconstruction. Amer. J. Sport Med. 1997; 25: 41-47. 